\title{
The Role of CXCL12 in Kidney Diseases: A Friend or Foe?
}

\author{
Anni Song Anni Jiang Wei Xiong Chun Zhang \\ Department of Nephrology, Union Hospital, Tongji Medical College, Huazhong University of Science and \\ Technology, Wuhan, China
}

\section{Keywords}

CXCL12 · CXCR4 · Acute kidney injury · Diabetic kidney disease $\cdot$ Lupus nephritis

\begin{abstract}
Background: Chemokines are a family of proteins mainly mediating the homing and migration of various cells. The CXC chemokine CXCL12 is a member of low-weight-molecular chemokines. In the kidney, CXCL12 is pivotal for renal development and exerts a modulatory effect in kidney diseases under different etiologic settings by binding with CXC chemokine receptor 4 (CXCR4) or CXC chemokine receptor 7 (CXCR7). Besides, CXCL12 also exerts homeostasis influence in diverse physical conditions and various pathological situations. Thus, we conclude the complicated relationship between CXCL12 and kidney diseases in this review. Summary: In renal development, CXCL12 contributes a lot to nephrogenesis and the formation of renal vasculature via correlating with CXCR4. CXCL12 also plays an essential role in renal recovery from acute kidney injury. However, the CXCL12/CXCR4 axis plays a dual regulatory role in the initiation and development of diabetic kidney disease as well as chronic allogeneic nephropathy after kidney transplanta-
\end{abstract}

C 2021 The Author(s)

Published by S. Karger AG, Basel

This is an Open Access article licensed under the Creative Commons Attribution-NonCommercial-4.0 International License (CC BY-NC) (http://www.karger.com/Services/OpenAccessLicense), applicable to the online version of the article only. Usage and distribution for commercial purposes requires written permission. tion through dialectical consideration. Additionally, the CXCL12/CXCR4 link is considered as a new risk factor for lupus nephritis and renal cell carcinoma. Key Messages: Plenty of studies have presented the influence of CXCL12 and the relation with corresponding receptors in diverse biological and pathological statuses. Simultaneously, some drugs and antagonists targeting CXCL12/CXCR4 axis effectively treat various kidney diseases. However, more researches are needed to explore thorough influence and mechanisms, providing more cues for clinical treatments.

(c) 2021 The Author(s)

Published by S. Karger AG, Basel

\section{Introduction}

CXCL12 was initially described in 1994 as pre-B cell growth-stimulating factor and was then named as stromal cell-derived factor-1 (SDF-1) in 1996 due to its continuous expression in bone marrow stromal cells $[1,2]$. According to differential mRNA splicing, CXCL12 was identified as 6 different splice variants including CXCL12 $\alpha$ to $\phi$ in humans while 3 variants (CXCL12 $\alpha$ to $\gamma$ ) were confirmed in mice $[3,4]$. As a member of chemokines, CXCL12 usually fulfills biological and pathological func-
Correspondence to:

Chun Zhang, drzhangchun @ hust.edu.cn 
tions by binding with corresponding receptors. CXC chemokine receptor 4 (CXCR4) has ever been regarded as a specific receptor of CXCL12 over a long period [5]. Recently, atypical chemokine receptor 3 (ACKR3), also known as CXC chemokine receptor 7 (CXCR7), has also been recognized as another vital receptor of CXCL12 [6]. As a member of homeostasis chemokines, which are composed of a large family of low-molecular-weight proteins, CXCL12 mainly enhances migration and adhesion of hematopoietic progenitor, stem cells, endothelial cells (ECs), and leukocytes. It is well recognized that CXCL12 plays a crucial role in diverse physical processes, including embryogenesis, organogenesis, and angiogenesis, while it also exerts crucial influence in various pathological situations, such as inflammation, organ injury regeneration, tumor invasion, and metastasis $[7,8]$.

It is well thought that CXCL12 contributes a lot to renal development, especially in renal vasculature formation [9]. It is also reported that CXCL12 is produced by a majority of renal cells to attract target cells and induce cell interactions via various signaling pathways $[10,11]$. In addition, there is accumulating evidence showing that CXCL12 is involved in various kidney diseases, such as acute kidney injury (AKI), diabetic kidney disease (DKD), lupus nephritis, kidney transplantation, and renal cell carcinoma (RCC). However, the underlying mechanisms are still unclear.

A further understanding of the interplay between CXCL12 and kidney diseases is beneficial to explore novel targets for disease precaution and treatment. In this review, we display the updated knowledge of the role of CXCL12 in renal development as well as the association with renal injury. Since the pathologic relationships among them are dissimilar in different etiologic situations, we willillustrate them with regard to theclassification of kidney diseases.

\section{The Role of CXCL12 in Nephrogenesis}

It is well known that CXCL12 plays a pivotal role in angiogenesis and organogenesis [8]. When the sight is focused on renal development, the CXCL12/CXCR4 axis also contributes a lot. Takabatake et al. [11] detected that adjacent parts of the podocytes also expressed CXCL12 in addition to stromal cells during glomerular development. As for CXCR4, it is consistently presented in the nephrogenic zone during embryogenesis. Precisely, Gröne et al. [10] exhibited that CXCR4 was strongly expressed in the ureteric bud and pretubular aggregates at the early em-

CXCL12 in Kidney Diseases: An Update bryonic stage while its expression diverted to cap mesenchyme instead of ureteric buds at a later stage. Notably, Tachibana et al. [12] found that mice lacking CXCL12 or CXCR4 easily underwent perinatal death. Moreover, CXCL12-secreting cells always surround CXCR4-positive cells in the nephrogenic zone, indicating stromal cells and podocytes mediate signaling transduction in the developing nephrons to promote nephrogenesis [11].

When it comes to mature glomeruli, CXCL12 is expressed in stromal cells as well as podocytes while CXCR4 is expressed in most ECs $[11,13]$. Thus, CXCL12 generated by podocytes and stromal cells may regulate survival signals or cell proliferation by interacting with CXCR4 on glomerular endothelial stalks. However, the precise mechanisms underlying the CXCL12/CXCR4 axis accelerating glomerular loop formation remain unclear. Takabatake et al. [11] revealed that both p44/42 mitogen-activated protein kinase (MAPK) and Akt pathways were activated in human umbilical vein ECs stimulated with recombinant CXCL12. Additionally, CXCL12 produced by podocytes could increase the level of CXCR4 in glomerular ECs. At the same time, Romoli et al. [14] observed that some interlobular arteries and afferent arterioles expressed simultaneously both CXCL12 and CXCR4, which supported that the CXCL12/CXCR4 axis improved angiogenic process via an autocrine pathway.

In conclusion, CXCL12 contributes a lot to nephrogenesis by binding with CXCR4, especially in the development of renal vasculature. However, the precise molecular mechanisms have not yet been fully understood, more efforts are needed to explore the underlying mechanisms.

\section{The Interplay between CXCL12 and Kidney Diseases}

\section{CXCL12 and Acute Kidney Injury}

$\mathrm{AKI}$ is defined as a sharp decline of renal function during a short period under different pathological settings. It is thought to be an independent risk parameter accounting for morbidity and mortality of hospitalized patients, especially patients in the intensive care unit [15]. There have been significant efforts to explore the cause of AKI, in which ischemia-reperfusion injury (IRI) is believed to be a critical issue $[16,17]$. Thereupon, IRI-induced defects in renal vascular integrity significantly affect renal regeneration. In addition, renal tubule is also sensitive to ischemic stress, ultimately leading to tubular necrosis. Moreover, inflammation infiltration is also a key pathophysiologic process during AKI. There is a consensus that 


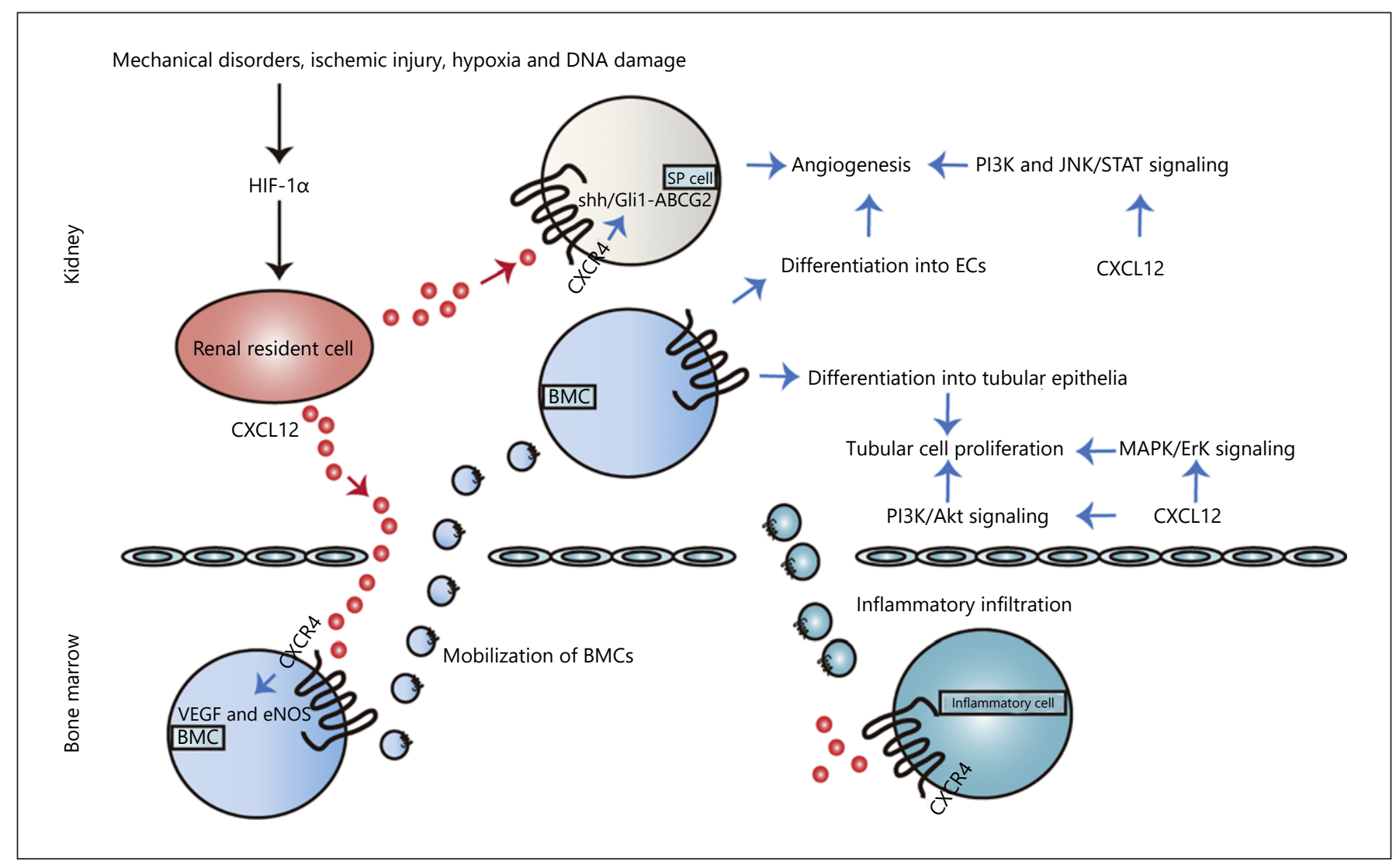

Fig. 1. Correlation between CXCL12 and AKI. Various pathological stimuli induce HIF-1a, which enhances the CXCL12/CXCR4 axis, ultimately improving AKI regeneration. On the one hand, the CXCL12/CXCR4 axis promotes angiogenesis by VEGF and eNOSmediated BMCs differentiation into ECs, SP cells-initiated recovery via Shh/Gli 1-ABCG2 pathway. In addition, diverse signaling pathways are also involved, such as PI3K and JNK/STAT signaling pathways. Additionally, the CXCL12/CXCR4 axis also increases

CXCL12 plays an essential role in the renal recovery from AKI as we delineate in the following section (shown in Fig. 1).

The renal capillary network repair requires the involvement of diverse cells. Among them, the differentiation of bone marrow-derived cells (BMCs) including EPCs and HSCs into vascular ECs and smooth muscle cells is essential for this process [18]. Tögel et al. [19] observed that CXCL12 showed an elevated level in the kidney after IRI-induced AKI along with decreased expression in the bone marrow, thus resulting in the reversal gradient between bone marrow and kidney. Then BMCs with increasing CXCR4 were modulated into the circulation and migrated to the injury kidney attracted by CXCL12-labeled cells. Subsequently, CXCL12 may tubular cell proliferation due to BMCs differentiation into the tubular epithelia and diverse pathways activation, such as PI3K/Akt and MAPK/Erk signaling pathways. Furthermore, CXCL12-induced inflammatory infiltration including neutrophils infiltration, $\mathrm{T}$-cell depletion, and macrophage initiation is also involved in AKI. AKI, acute kidney injury; EC, endothelial cell; BMC, bone marrow-derived cell; eNOS, endothelial nitric oxide synthase; SP, side population; TEC, tubular epithelia cell. participate in BMCs differentiation into ECs by enhancing vessel endothelial growth factor (VEGF) expression [20]. Of note, both CXCL12 and VEGF could be regulated by hypoxia-inducible factor-1a (HIF-1 $\alpha$ ) induced by various pathological settings, such as mechanical disorders, ischemic injury, hypoxia, and DNA damage. Besides, HIF-1 $\alpha$ also improved CXCR4 generation, which accelerated vessel reconstitution by increasing VEGF and endothelial nitric oxide synthase (eNOS) secretion [21].

Moreover, Ge et al. [22] focused on the role of side population (SP) cells in renal injury repair. SP cells harboring stem cell-like characteristics exist in diverse tissues, including kidney. Present studies manifested the marked upregulation of CXCL12 and CXCR4 in SP cells 
upon IRI. Additionally, CXCL12 increased ABCG2 expression in SP cells simulated with IRI through Shh-Gli1 signaling [22]. Meanwhile, CXCL12/CXCR4 improved $\mathrm{SP}$ cells chemotaxis and paracrine actions. Furthermore, blocking CXCR4 with the corresponding antagonists counteracted SP cell-initiated renal recovery. Overall, the CXCL12/CXCR4 axis may participate in SP cells-mediated renoprotection in IRI-induced AKI via Shh/Gli1-ABCG2 pathway.

Taking the characteristics of CXCR4 as a member of GPCRs into account, CXCL12 may activate transcription factors to regulate renal cell proliferation, apoptosis and ultimately promote angiogenesis by inducing intracellular downstream cascades, such as intracellular ras, phosphatidylinositol 3-kinase (PI3K), Janus kinase/signal transducer and activators of transcription (JNK/STAT) signaling [23].

It has been well described that dipeptidyl peptidase (DPP)-4 inhibitors serve as antidiabetic drugs [24, 25]. Recently, Kamel et al. [21] identified the renoprotective effect of DPP-4 inhibitor saxagliptin in IRI-induced AKI. In IRI rats, saxagliptin sustained CXCL12 expression as well as upregulated the level of CXCR4 to trigger angiogenesis via the modulation and homing of EPCs [21]. Additionally, saxagliptin also stimulated kidney injury molecule-1 (Kim-1)/STAT-3/HIF-1 $\alpha$ /VEGF pathway, accompanied by levels of GLP-1/cAMP [21]. Accordingly, saxagliptin-mediated upregulation of CXCL12/CXCR4 axis may be an outcome of Kim-1/STAT-3/HIF-1a/ VEGF pathway coupling with the enhanced expressions of GLP-1/cAMP.

In addition to vascular integrity, renal tubular injury reconstitution also needs more attentions, which depends to a large extent on BMCs homing to the kidney and harboring a phenotype of tubular epithelia. In healthy human kidneys, Lotan et al. [26] discovered that strong immunoreactivity of anti-CXCL12 antibodies could be detected in renal tubular cells. In a model of AKI, renal epithelial cells secreted CXCL12 in response to hypoxia, while hepatocyte growth factor (HGF) was produced in renal interstitium [18]. Both CXCL12 and HGF are essential for migration of BMCs to injured tubules as well as differentiation to the renal tubular epithelia cells (TECs). Neutralizing HGF in AKI mice resulted in renal hypoxia and CXCL12 upregulation, inhibiting tubular cell proliferation and BMCs recruitment [27]. On the other hand, CXCL12 mediated MAPK/Erk kinase pathway by interacting with upregulated CXCR4 to enhance the proliferation of renal epithelial cells, as well as PI3K/Akt signaling [28].

CXCL12 in Kidney Diseases: An Update
Furthermore, it is worthy to explore whether CXCL12 participates in recruiting inflammatory cells during AKI initiation and progression due to the CXCR4 presence on the surface of various inflammation cells. Actually, little is known about the role of CXCL12 in AKI-associated inflammation infiltration. Stokman et al. [28] reported that CXCL12 antisense administration amplified an intrarenal inflammatory reaction during IRI, different from nonkidney inflammation infiltration including LPS-induced lung injury. It was possible that the increased number of apoptotic TECs improved the demand for neutrophils to migrate to the kidney to clear injured cells [28]. However, Tögel et al. [19] found that T-cell repulsion was induced when the concentration of renal CXCL12 after IRI reached the concentration detected in the bone marrow, thereby exerting an anti-inflammatory effect. Even more to the point, Wan et al. [23] suggested that renal CXCL12 accumulated in response to IRI and was significantly upregulated upon macrophage depletion, indicating that the initial inflammatory response for kidney injury likely determined CXCL12 activation in turn.

Taken together, CXCL12/CXCR4 axis contributes to AKI regeneration by promoting angiogenesis and tubular cell proliferation via various mechanisms. Moreover, the interaction between CXCL12 and inflammatory response including neutrophils infiltration, T-cell depletion, and macrophage initiation is also involved in AKI.

\section{CXCL12 and Diabetic Kidney Disease}

As a well-known complication of diabetes, DKD has been one of the most common causes of end-stage kidney disease. There have been lots of investigations to explore the mechanisms of $\mathrm{DKD}$, podocyte injury, and mesangial expansion are thought to be significant events $[29,30]$. CXCL12 plays an essential regulatory role in the initiation and development of DKD, however, whether it is beneficial or harmful requires dialectical consideration as we sketch in the following part (shown in Fig. 2) $[31,32]$.

A growing body of data suggests that CXCL12 exerts a renoprotective effect in DKD by binding with CXCR4. The CXCL12/CXCR4 axis maintains the glomerular structure by promoting renal podocyte survival, reducing mesangial expansion and tubular epithelial cell death [31, 33]. Takashima et al. [31] reported that CXCR4 antagonists resulted in podocyte disorder by inhibiting CXCL12/ CXCR4 interaction in DKD mice. Besides, CXCR4 blockade also promoted hyperglycemia-stimulated renal TEC death by increasing the level of Bcl-2-related death promoter (BAD) and preventing Akt phosphorylation [33]. Moreover, Zhang et al. [34] reported that CXCL12/ 


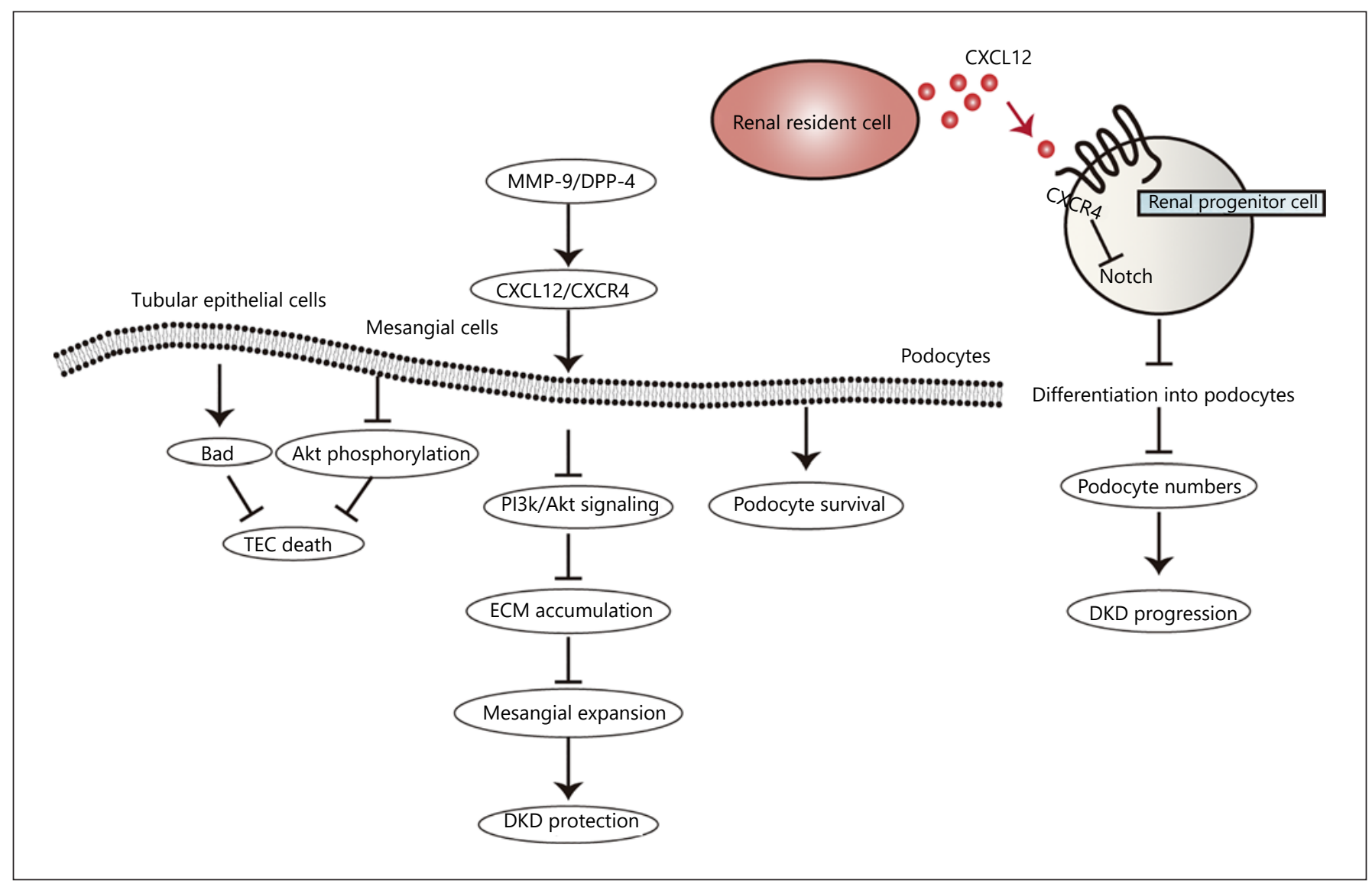

Fig. 2. The CXCL12/CXCR4 axis-mediated signaling network in DKD. The CXCL12/CXCR4 axis plays a dual regulatory role in DKD. On the one hand, CXCL12/CXCR4 leads to TEC decrease, mesangial expansion reduction, and podocyte survival, which contribute to diabetic renoprotection. TEC death is decreased via BAD and Akt phosphorylation while fibronectin expression and ECM accumula-

CXCR4 correlation inhibited fibronectin expression and extracellular matrix accumulation through PI3K/Akt signal pathway in high glucose-induced mesangial cells.

Siddiqi et al. [33] elucidated that CXCL12 expression was enhanced accompanied by the upregulation of matrix metalloproteinase-9 (MMP-9) in both DKD patients and diabetic rats. Due to the effect of cleaving CXCL12, MMP-9 contributed to reducing the phosphorylation of CXCR4 and eliminating the phosphorylation of downstream Akt in turn [35]. Thus, MMP-9-mediated CXCL12/CXCR4 inactivation depresses its renal protection in DKD.

Dipeptidyl peptidase-4 (DPP-4) is another cleavage enzyme of CXCL12 [36], its inhibitors can further increase the expression of CXCL12 in glomerular and medullary tissues [31]. Fujita et al. [37] treated patients in the tion are inhibited through PI3K/Akt signal pathway, reducing mesangial expansion. On the other hand, the CXCL12/CXCR4 axis mediates podocyte reduction by reducing podocyte progenitor cell differentiation into podocytes via inhibiting Notch signal transduction, resulting in DKD progression. DKD, diabetic kidney disease; TEC, tubular epithelia cell; ECM, extracellular matrix.

early DKD stage with DPP-4 inhibitors based on angiotensin II type 1 receptor blockade, they found that the therapy prevented DKD progression along with renal oxidative stress and proteinuria reduction by activating the CXCL12-cAMP pathway. Therefore, DPP-4 inhibitors can sustain glomerular podocytes via the antioxidant and antifibrotic effects of CXCL12 [31].

Nowadays, a series of investigations report that blocking the CXCL12/CXCR4 axis exerts a therapeutic effect in DKD clinical treatment $[32,38]$. The CXCL12/CXCR4 axis is not only beneficial to renal regeneration, but it is also indeed related to DKD progression. Sayyed et al. [38] observed that CXCL12 produced mainly by podocytes potentiated proteinuria and glomerulosclerosis in type 2 diabetic $\mathrm{db} / \mathrm{db}$ mice. Subsequently, they found that temporarily blocking CXCL12 could effectively prevent the 
progression of glomerulosclerosis and albuminuria. Moreover, CXCL12 inhibition is also associated with an increase in the number of podocytes, resulting from podocyte death reduction and podocyte regeneration promotion. Also, Darisipudi et al. [32] found that dual chemokine blockade could also have an additional preventive effect on the progression of DKD by double blocking CCL2 and CXCL12.

So how does the CXCL12/CXCR4 axis mediate the progression of $\mathrm{DKD}$ ? It is well recognized that the subpopulation of parietal epithelial cells in the glomeruli represents podocyte progenitor cells and can migrate and differentiate into podocytes [31, 37]. CXCL12 inhibits the induced maturation of renal progenitor cells into podocytes by downregulating renin expression, while CXCL12 blocking can enhance the differentiation of renal progenitor cells into podocyte lineages, thus enhancing podocyte regeneration $[32,39]$. There are also some relatively innovative viewpoints, Romoli et al. [14] believed that CXCL12 expressed by podocytes reduced the activation of podocyte progenitor cells and maintained their quiescence by inhibiting Notch signal transduction, accordingly restricting their regeneration into lost podocytes. Therefore, CXCL12 blockade strengthened podocyte regeneration powerfully in cortical nephrons.

Due to the expression of CXCR4 on the surface of diverse inflammation cells including neutrophils, lymphocyte T, and B cells [40], it is plausible that upregulated expression of the receptor may contribute to the renal inflammation in $\mathrm{DKD}$ progression. Interestingly, there is little of literatures reporting the effect of CXCL12 on inflammation in DKD. Darisipudi et al. [32] found that CCL2 but not CXCL12 blockade decreased glomerular $\mathrm{CD} 45^{+}$leukocyte numbers in $\mathrm{db} / \mathrm{db}$ mice. Meanwhile, Sayyed et al. [38] indicated that the numbers of renal macrophages was not affected by CXCL12 inhibition in $\mathrm{db} /$ $\mathrm{db}$ mice. However, an alteration with upregulated phenotype expressions of both M1 and M2 markers is detected. Notably, these researchers had opposite opinions on the effect of CXCL12 on DKD progression, which making it convincing that CXCL12 stimulation exerts no effect on inflammatory reaction in $\mathrm{DKD}$ at present.

In conclusion, CXCL12/CXCR4 axis exerts a dual effect on DKD progression. The exact relationship between CXCL12/CXCR4 and DKD deserves further attentions. There is still a long way to go before CXCL12 becoming an ideal therapeutic target considering the fact that CXCL12/CXCR4 has a complex effect on DKD.

\section{CXCL12 and Lupus Nephritis}

Inflammatory infiltration and autoantibodies deposits are cardinal to the pathogenesis of systemic lupus erythematosus-mediated organ perturbation, specifically lupus nephritis [41]. Although CXCL12 is defined as a homeostasis chemokine, several investigations showed that CXCL12 might be a risk factor for the progression of lupus nephritis (shown in see online suppl. Fig. 1; see www. karger.com/doi/10.1159/000514913 for all online suppl. material) [42-44].

Antibody-secreting cells (ASCs), including plasmablasts and memory plasma cells, are essential for lupus nephritis. In NZB/W mice, Balabanian et al. [43] confirmed that the upregulation of CXCL12 depended largely on podocytes in glomeruli. Simultaneously, CXCR4positive ASCs were attracted to the kidney, leading to autoantibodies deposits. In addition to ASCs, other lymphocytes, including $\mathrm{T}$ cells, peritoneal $\mathrm{B} 1 \mathrm{a}$ (PerB1a) lymphocytes, were also activated and modulated to renal tissues via CXCL12/CXCR4 axis [43]. Furthermore, Badr et al. [44] proposed that B cells involved in lupus nephritis presented a marked upregulation in responsiveness to CXCL12 via PI3K/AKT, NF- $\kappa B$, and ERK signaling excessive activation. Meanwhile, the correlation between CXCR4 and CXCL12 also triggered MAPK and JAK/ STAT signaling, ultimately initiating aberrant cellular issues [41]. Interestingly, CXCR4 enhancement in podocytes is consistent and even strengthened by CXCL12. Then the CXCL12/CXCR4 axis may activate NADPHoxidase via an autocrine pathway, thereupon resulting in reactive oxygen stress accumulation, consequently attacking the glomeruli of NZB/W mice [43].

In contrast, both CXCL12 neutralization and CXCR4 antagonists can restore renal function and attenuate lupus nephritis damage. Balabanian et al. [43] illustrated that CXCL12 neutralization prevented proteinuria and death in NZB/W mice along with decreased numbers of PerB1a lymphocytes as well as suppression of T lymphocytes activation. Additionally, Cheng et al. [45] elucidated that long-term CXCR4 blocker treatment downregulated the level of autoantibodies uncoupling with inhibition of the inflammatory process in lupus nephritis. Thus, the CXCL12/CXCR4 axis results in lupus nephritis progression via ACSs-mediated autoantibodies deposits as well as $\mathrm{T}$ cell and PerB1a lymphocytes infiltration in diverse signaling pathways.

\section{CXCL12 and Kidney Transplantation}

Kidney transplantation is the effective treatment for ESRD patients. However, kidney transplant recipients are 
likely to develop chronic allogeneic nephropathy (CAN), resulting in graft loss in the long run. Tubular atrophy and interstitial fibrosis are critical pathological events contributing to CAN $[46,47]$, while IRI and inflammation infiltration are also proved to account for CAN (shown in online suppl. Fig. 2) [48]. Hoffmann et al. [49] presented that the expression of CXCL12 in CAN recipients was significantly increased compared with healthy transplant kidneys. CXCL12/CXCR4 axis is considered to exert complex effects on renal grafts according to the degree of renal damage and the level of CXCL12/CXCR4 [48].

Moreover, it is reported that the elevation of CXCL12 expressions potentiates renal allografts fibrosis. Thus, CXCL12/CXCR4 pathway activation can promote CAN progression, while inhibition of the relation can effectively attenuate renal allograft fibrosis advancement, ultimately improving CAN [50, 51]. Zou et al. [48] also confirmed that neutralizing CXCR4 inhibited the fibrotic expression and downregulated transforming growth factor- $\beta 1$, thus reducing interstitial fibrosis in long-term survival allografts.

It is also observed that diverse immune cells and downstream signal pathways are involved in CAN [48]. Activated $\mathrm{T}$-cell recruitment to renal tubules is a significant feature of renal transplant rejection. Al-Hamidi et al. [52] indicated that chemokines including CXCL12 directly enhanced $\mathrm{T}$ cell-mediated epithelial-mesenchymal transition during chronic allogeneic renal failure by promoting adhesive contact between activated $\mathrm{T}$ cells and renal TECs. Tang et al. [50] also described that CXCL12/ CXCR4 induced epithelial-mesenchymal transition by activating the $\mathrm{Wnt} / \beta$-catenin pathway, leading to CAN progression.

Besides, Gao and Huan's [53] team also discovered that anti-CXCL12 antibodies could reduce IRI and chronic rejection injury, preserve renal function, and even delay CAN procession. The downregulation of CXCL12 expression has a renoprotective effect in IRI-induced renal transplantation injury.

On the other hand, Cao et al. [54] reported that CXCL2/ CXCR4 enhancement promoted bone marrow mesenchymal stem cells (MSCs) homing to transplanted kidneys, thus preventing IRI-induced acute/chronic rejection and maintaining renal function. CXCL12 is an essential medium for CXCR4-positive cells to home and migrate to the kidney [40]. Notably, Ratajczak et al. [55] found that the concentration of CXCL12 was relatively low, insufficient to induce stem cells to flow out of the bone marrow during the perioperative period of kidney transplantation, which suggested that the potential CXCL12 was not yet released from the transplanted kidney to the periphery in the early stage of renal transplant reperfusion. As IRI progression, the expression of CXCL12 appears to be upregulated [40, 49], which mediates the recruitment and attachment of CXCR4-positive cells, such as HSCs and MSCs, to the transplanted kidney [40]. MSCs treatment indeed alleviates IRI in the graft along with the majority of transplanted cells located in the kidney and CXCL12/CXCR4 tight correlation [54]. CXCL12/CXCR4 regulates MSCs homing and implantation in transplanted kidneys.

Furthermore, it has been reported that donor genetic CXCL12 polymorphism affects the outcome of renal allografts [56]. Both Lee et al. [57] and Wang et al's. [58] teams proposed that AA/AG genotype and an allele of CXCL12 had adverse effects on the endpoint of allograft kidney transplantation. Therefore, genetic effects from donors should be carefully considered in order to select appropriate donors in kidney transplantation. Conclusively, the CXCL12/CXCR4 axis is an essential regulator for the survival of renal allografts in transplant patients.

Based on the available data from plenty of researches, it appeared unclear whether CXCL12 predominantly protects from CAN or whether it contributes to progression of CAN (e.g., by promoting inflammation infiltration and leading to renal allografts fibrosis as well as IRI), the relationship between CXCL12/CXCR4 axis and CAN deserves further researches.

\section{CXCL12 and Renal Cell Carcinoma}

Clear cell RCC (cc-RCC) is the most common subtype of RCC, mainly characterized by the inactivation of the von Hippel-Lindau (VHL). VHL can induce various target proteins degradation, including HIF-1a, a key transcription factor for CXCL12 gene expression in RCC [59]. Struckmann et al. [60] demonstrated that VHL dysfunction led to the accumulation of HIF-1a coupled with increased expressions of CXCL12/CXCR4 in cc-RCC, ultimately leading to the imbalance of cell growth and angiogenesis (shown in online suppl. Fig. 3).

Both Wu et al [61] and Wang et al's [62] teams showed that CXCL12 was one of the central potential target genes and biomarkers of cc-RCC, contributing to deciphering the molecular mechanisms of cc-RCC occurrence as well as being used as a diagnostic biomarker and therapeutic target for cc-RCC. The genetic polymorphisms of CXCL12/CXCR4 are related to the increased sensitivity of Chinese to RCC, indicating a new risk factor for RCC in the Chinese population [63]. 
Gahan et al [64] and Schrader et al. [65] indicated that compared with normal kidney tissues, the expressions of CXCL12, CXCR4, and CXCR7 in RCC tissues were upregulated. Simultaneously, Wang et al. [66] and Wehler et al's. [67] teams proved that the elevations were associated with poor overall survival and relapse-free survival of RCC. Rasti et al. [68] also discovered that the expression of CXCR4 was positively correlated with the grade, stage, and microvascular invasion of RCC. Moreover, in the view of Tang et al. [69], the expression of CXCR4 was relatively stronger in RCC patients with lower Fuhrman grade.

As known, RCC has the characteristic of organ-specific metastasis. The CXCL12/CXCR4 axis also provides RCC with a strong ability to regulate cell cycle and inhibit apoptosis, as well as invade the tissue barrier and migrate to other organs $[59,60,70]$. Interestingly, Wang et al. [66] detected that the level of CXCL12 in surrounding tissues was higher than that in RCC tissues, suggesting that a possible metastasis channel was constructed between cancer tissues and surrounding normal tissues and organs via the concentration gradient of CXCL12. Besides, they also showed that the expression of CXCR4 in metastatic foci of RCC was higher than that of RCC in situ. At the same time, CXCR4 was mainly localized in cytoplasm and nucleus in metastatic RCC instead of the cell membrane in primary RCC, indicating that metastatic ability of RCC was related to the high expression and nuclear localization of CXCR4 [71, 72].

Furthermore, Jones et al. [73] raised that the CXCL12/ CXCR4 axis was also involved in triggering the adhesion of RCC cells to vascular ECs and subendothelial matrix, contributing to RCC metastasis. Notably, CXCR4 expressed by RCC cells mediates the chemical inducement of CXCL12 secreted by the target organs [74], CXCL12 induces internalization of surface CXCR4 in turn [75]. Besides, the study constructed by Gassenmaier et al. [76] proved that CXCR4 could also predict the survival time of nonmetastatic RCC patients. Gahan et al. [64] also established a prospective study reporting that $\mathrm{CXCL} 12^{+} \mathrm{CXCR} 7^{+}$had approximately $80 \%$ accuracy in predicting cc-RCC metastasis.

In addition, Pan et al. [59] found that the treatment of specific anti-CXCL12 antibody significantly reduced the transfer of RCC to target organs in the in situ RCC model. Wang et al. [74] also targeted CXCR4 to inhibit the metastatic process of RCC, leading to RCC cell apoptosis. It can be seen that the regulation of CXCR4/CXCL12/ CXCR7 biological axis may be a potential therapeutic target for RCC. As a result, CXCL12/CXCR4 axis leads to RCC poor survival by promoting tumor growth and metastasis via various mechanisms, which provides a promising target regarding RCC treatment.

\section{Conclusion}

CXCL12 belongs to a large family of small protein chemokines and its interplay with receptors including CXCR4 and CXCR7 is involved in various biological and pathological situations. In the kidney, the CXCL12/ CXCR4 interaction advances renal development, especially vasculature formation. Notably, CXCL12 plays a dual role in renal diseases of different etiologic settings. CXCL12/CXCR4 axis mediates BMCs activation and homing to the defected kidney, which results in renal regeneration or accelerates disease advancement. The data aforementioned above have postulated that DPP-4 inhibitors and CXCL12 neutralization may be promising therapeutic ideas through sustaining and dampening CXCL12 expression, respectively. However, no clinical trials have been carried out to identify the role of CXCL12 in renal diseases. The underlying molecular mechanisms about the interaction between CXCL12 and kidney injury also lack thorough investigations. Accordingly, current data and conclusions may provide some clues for renal disease treatments and novel drug exploitation. Simultaneously, we still need to pay more efforts to explore the nature of CXCL2 in renal diseases via numerous fundamental and clinical studies.

\section{Conflict of Interest Statement}

The authors have no conflicts of interest to disclose and the results in this paper have not been published previously.

\section{Funding Sources}

This work was financially supported by the National Natural Science Foundation of China (81974096, 81961138007, 81974097, $81873602,81770711,81700603,81522010$, and 81470964), the National Key Research and Development Program (2020YFC0845800, 2020YFC0844700, 2018YFC1314000), and the Program for HUST Academic Frontier Youth Team (2017QYTD20).

\section{Author Contributions}

Chun Zhang conceptualized the review. Anni Song and Anni Jiang wrote a draft of the review and equally contributed to this paper. Chun Zhang, Wei Xiong, and Anni Song revised the paper. The figures were drawn by Anni Song and Anni Jiang. Chun Zhang performed the final edits. 


\section{References}

1 Bleul CC, Fuhlbrigge RC, Casasnovas JM, Aiuti A, Springer TA. A highly efficacious lymphocyte chemoattractant, stromal cell-derived factor 1 (SDF-1). J Exp Med. 1996 Sep;184(3): 1101-9.

2 Nagasawa T, Kikutani H, Kishimoto T. Molecular cloning and structure of a pre-B-cell growth-stimulating factor. Proc Natl Acad Sci USA. 1994 Mar;91(6):2305-9.

3 Shirozu M, Nakano T, Inazawa J, Tashiro K, Tada H, Shinohara H, et al. Structure and chromosomal localization of the human stromal cell-derived factor 1 (SDF-1) gene. Genomics. 1995 Aug;28(3):495-500

4 Yu L, Cecil J, Peng SB, Schrementi J, Kovacevic S, Paul D, et al. Identification and expression of novel isoforms of human stromal cell-derived factor 1. Gene. 2006 Jun;374:174-9.

5 Bachelerie F, Ben-Baruch A, Burkhardt AM, Combadiere C, Farber JM, Graham GJ, et al. International union of basic and clinical pharmacology. [corrected]. LXXXIX. Update on the extended family of chemokine receptors and introducing a new nomenclature for atypical chemokine receptors. Pharmacol Rev. 2013 Nov;66(1):1-79.

6 Sierro F, Biben C, Martínez-Muñoz L, Mellado M, Ransohoff RM, Li M, et al. Disrupted cardiac development but normal hematopoiesis in mice deficient in the second CXCL12/SDF-1 receptor, CXCR7. Proc Natl Acad Sci USA. 2007 Sep;104(37):14759-64.

7 Nagasawa T. A chemokine, SDF-1/PBSF, and its receptor, $\mathrm{CXC}$ chemokine receptor 4 , as mediators of hematopoiesis. Int J Hematol. 2000 Dec;72(4):408-11.

8 Ratajczak MZ, Zuba-Surma E, Kucia M, Reca R, Wojakowski W, Ratajczak J. The pleiotropic effects of the SDF-1-CXCR4 axis in organogenesis, regeneration and tumorigenesis. Leukemia. 2006 Nov;20(11):1915-24.

9 Janssens R, Struyf S, Proost P. The unique structural and functional features of CXCL12. Cell Mol Immunol. 2018 Apr;15(4):299-311.

10 Gröne HJ, Cohen CD, Gröne E, Schmidt C, Kretzler M, Schlöndorff D, et al. Spatial and temporally restricted expression of chemokines and chemokine receptors in the developing human kidney. J Am Soc Nephrol. 2002 Apr; 13(4):957-67.

11 Takabatake Y, Sugiyama T, Kohara H, Matsusaka T, Kurihara H, Koni PA, et al. The CXCL12 (SDF-1)/CXCR4 axis is essential for the development of renal vasculature. J Am Soc Nephrol. 2009 Aug;20(8):1714-23.

12 Tachibana K, Hirota S, Iizasa H, Yoshida H, Kawabata K, Kataoka Y, et al. The chemokine receptor CXCR4 is essential for vascularization of the gastrointestinal tract. Nature. 1998 Jun; 393(6685):591-4.

13 Yakulov TA, Todkar AP, Slanchev K, Wiegel J, Bona A, Groß M, et al. CXCL12 and MYC control energy metabolism to support adaptive responses after kidney injury. Nat Commun. 2018 Sep;9(1):3660.
14 Romoli S, Angelotti ML, Antonelli G, Kumar Vr S, Mulay SR, Desai J, et al. CXCL12 blockade preferentially regenerates lost podocytes in cortical nephrons by targeting an intrinsic podocyte-progenitor feedback mechanism. Kidney Int. 2018 Dec;94(6):1111-26.

15 Goldberg R, Dennen P. Long-term outcomes of acute kidney injury. Adv Chronic Kidney Dis. 2008 Jul;15(3):297-307.

16 Hussein AM, Barakat N, Awadalla A, Gabr MM, Khater S, Harraz AM, et al. Modulation of renal ischemia/reperfusion in rats by a combination of ischemic preconditioning and adipose-derived mesenchymal stem cells (ADMSCs). Can J Physiol Pharmacol. 2016 Sep;94(9): 936-46.

17 Sharfuddin AA, Molitoris BA. Pathophysiology of ischemic acute kidney injury. Nat Rev Nephrol. 2011 Apr;7(4):189-200.

18 Ohnishi H, Mizuno S, Mizuno-Horikawa Y, Kato T. Stromal cell-derived factor-1 (SDF1)dependent recruitment of bone marrow-derived renal endothelium-like cells in a mouse model of acute kidney injury. J Vet Med Sci. 2015 Mar;77(3):313-9.

19 Tögel F, Isaac J, Hu Z, Weiss K, Westenfelder C. Renal SDF-1 signals mobilization and homing of CXCR4-positive cells to the kidney after ischemic injury. Kidney Int. 2005 May;67(5): 1772-84.

20 Bo CJ, Chen B, Jia RP, Zhu JG, Cao P, Liu H, et al. Effects of ischemic preconditioning in the late phase on homing of endothelial progenitor cells in renal ischemia/reperfusion injury. Transplant Proc. 2013 Mar;45(2):511-6.

21 Kamel NM, EI Fattah MA, EI-Abhar HS, Abdallah DM. Novel repair mechanisms in a renal ischemia/reperfusion model: subsequent saxagliptin treatment modulates the pro-angiogenic GLP-1/cAMP/VEGF, ANP/eNOS/NO, SDF-1a/CXCR4, and Kim-1/STAT3/HIF-1 $\alpha$ / VEGF/eNOS pathways. Eur J Pharmacol. 2019 Oct;861:172620.

22 Ge G, Zhang H, Li R, Liu H. The function of SDF-1-CXCR4 axis in SP cells-mediated protective role for renal ischemia/reperfusion injury by SHH/GLI1-ABCG2 pathway. Shock. 2017 Feb;47(2):251-9.

23 Wan X, Xia WK, Gendoo Y, Chen W, Sun WJ, Sun D, et al. Upregulation of stromal cell-derived factor 1 (SDF-1) is associated with macrophage infiltration in renal ischemia-reperfusion injury. PLoS One. 2017 Feb;47(2):251-9.

24 Crepaldi G, Carruba M, Comaschi M, Del Prato S, Frajese G, Paolisso G, et al. Dipeptidyl peptidase 4 (DPP-4) inhibitors and their role in type 2 diabetes management. J Endocrinol Invest. 2007 Jul-Aug;30(7):610-4.

25 Rathmann W, Kostev K, Gruenberger JB, Dworak M, Bader G, Giani G, et al. Treatment persistence, hypoglycaemia and clinical outcomes in type 2 diabetes patients with dipeptidyl peptidase- 4 inhibitors and sulphonylureas: a primary care database analysis. Diabetes Obes Metab. 2013 Jan;15(1):55-61.
26 Lotan D, Sheinberg N, Kopolovic J, Dekel B. Expression of SDF-1/CXCR4 in injured human kidneys. Pediatr Nephrol. 2008 Jan;23(1):71-7.

27 Ohnishi H, Mizuno S, Nakamura T. Inhibition of tubular cell proliferation by neutralizing endogenous HGF leads to renal hypoxia and bone marrow-derived cell engraftment in acute renal failure. Am J Physiol Renal Physiol. 2008 Feb; 294(2):F326-35.

28 Stokman G, Stroo I, Claessen N, Teske GJ, Florquin S, Leemans JC. SDF-1 provides morphological and functional protection against renal ischaemia/reperfusion injury. Nephrol Dial Transplant. 2010 Dec;25(12):3852-9.

29 Barrera-Chimal J, Jaisser F. Pathophysiologic mechanisms in diabetic kidney disease: a focus on current and future therapeutic targets. Diabetes Obes Metab. 2020 Apr;22(Suppl 1):1631.

30 Palygin O, Spires D, Levchenko V, Bohovyk R, Fedoriuk M, Klemens CA, et al. Progression of diabetic kidney disease in T2DN rats. Am J Physiol Renal Physiol. 2019 Dec;317(6):F145061.

31 Takashima S, Fujita H, Fujishima H, Shimizu T, Sato T, Morii T, et al. Stromal cell-derived factor-1 is upregulated by dipeptidyl peptidase- 4 inhibition and has protective roles in progressive diabetic nephropathy. Kidney Int. 2016 Oct;90(4):783-96.

32 Darisipudi MN, Kulkarni OP, Sayyed SG, Ryu M, Migliorini A, Sagrinati C, et al. Dual blockade of the homeostatic chemokine CXCL12 and the proinflammatory chemokine CCL2 has additive protective effects on diabetic kidney disease. Am J Pathol. 2011 Jul;179(1):116-24.

33 Siddiqi FS, Chen LH, Advani SL, Thai K, Batchu SN, Alghamdi TA, et al. CXCR4 promotes renal tubular cell survival in male diabetic rats: implications for ligand inactivation in the human kidney. Endocrinology. 2015 Mar;156(3):1121-32.

34 Zhang D, Shao S, Shuai H, Ding Y, Shi W, Wang D, et al. SDF-1 $\alpha$ reduces fibronectin expression in rat mesangial cells induced by TGF- $\beta 1$ and high glucose through PI3K/Akt pathway. Exp Cell Res. 2013 Jul 15;319(12): 1796-803.

35 McQuibban GA, Butler GS, Gong JH, Bendall L, Power C, Clark-Lewis I, et al. Matrix metalloproteinase activity inactivates the $\mathrm{CXC}$ chemokine stromal cell-derived factor-1. J Biol Chem. 2001 Nov;276(47):43503-8.

36 Hocher B, Reichetzeder C, Alter ML. Renal and cardiac effects of DPP4 inhibitors--from preclinical development to clinical research. Kidney Blood Press Res. 2012;36(1):65-84.

37 Fujita H, Taniai H, Murayama H, Ohshiro H, Hayashi H, Sato S, et al. DPP-4 inhibition with alogliptin on top of angiotensin II type 1 receptor blockade ameliorates albuminuria via upregulation of SDF-1 $\alpha$ in type 2 diabetic patients with incipient nephropathy. Endocr J. 2014; 61(2):159-66 
38 Sayyed SG, Hägele H, Kulkarni OP, Endlich K, Segerer S, Eulberg D, et al. Podocytes produce homeostatic chemokine stromal cell-derived factor-1/CXCL12, which contributes to glomerulosclerosis, podocyte loss and albuminuria in a mouse model of type 2 diabetes. Diabetologia. 2009 Nov;52(11):2445-54.

39 Sagrinati C, Netti GS, Mazzinghi B, Lazzeri E, Liotta F, Frosali F, et al. Isolation and characterization of multipotent progenitor cells from the Bowman's capsule of adult human kidneys. J Am Soc Nephrol. 2006 Sep;17(9):2443-56.

40 Togel FE, Westenfelder C. Role of SDF-1 as a regulatory chemokine in renal regeneration after acute kidney injury. Kidney Int Suppl. 2011 Sep;1(3):87-9.

41 Fairhurst AM, Wandstrat AE, Wakeland EK Systemic lupus erythematosus: multiple immunological phenotypes in a complex genetic disease. Adv Immunol. 2006;92:1-69.

42 Wang W, Rangel-Moreno J, Owen T, Barnard J, Nevarez S, Ichikawa HT, et al. Long-term B cell depletion in murine lupus eliminates autoantibody-secreting cells and is associated with alterations in the kidney plasma cell niche. J Immunol. 2014 Apr;192(7):3011-20.

43 Balabanian K, Couderc J, Bouchet-Delbos L, Amara A, Berrebi D, Foussat A, et al. Role of the chemokine stromal cell-derived factor 1 in autoantibody production and nephritis in murine lupus. J Immunol. 2003 Mar;170(6):3392-400.

44 Badr G, Sayed A, Abdel-Maksoud MA, Mohamed AO, El-Amir A, Abdel-Ghaffar FA, et al. Infection of female BWF1 lupus mice with malaria parasite attenuates $\mathrm{B}$ cell autoreactivity by modulating the CXCL12/CXCR4 axis and its downstream signals PI3K/AKT, NFkB and ERK. PLoS One. 2015 Apr;10(4):e0125340.

45 Cheng Q, Khodadadi L, Taddeo A, Klotsche J, F Hoyer B, Radbruch A, et al. CXCR4-CXCL12 interaction is important for plasma cell homing and survival in NZB/W mice. Eur J Immunol. 2018 Jun;48(6):1020-9.

46 Solez K, Colvin RB, Racusen LC, Sis B, Halloran PF, Birk PE, et al. Banff'05 meeting report: differential diagnosis of chronic allograft injury and elimination of chronic allograft nephropathy (CAN). Am J Transplant. 2007 Mar;7(3) $518-26$.

47 Hara S. Banff 2013 update: pearls and pitfalls in transplant renal pathology. Nephrology. 2015 Jul;20(Suppl 2):2-8.

48 Zou XF, Gu JH, Cui ZL, Lu YW, Gu C. CXC chemokine receptor type 4 antagonism ameliorated allograft fibrosis in rat kidney transplant model. Exp Clin Transplant. 2017 Aug;15(4): $448-52$.

49 Hoffmann U, Banas B, Krüger B, Banas M, Bergler T, Böger C, et al. SDF-1 expression is elevated in chronic human renal allograft rejection. Clin Transplant. 2006 Nov-Dec;20(6):712-8.

50 Tang H, Xu Y, Zhang ZJ, Zeng S, Dong WB, Hu XP. SDF-1/CXCR4 induces epithelial mesenchymal transition through activation of the Wnt /beta catenin signaling pathway in rat chronic allograft nephropathy. Mol Med Rep. 2019 May;19(5):3696-706.
51 Xu Y, Zhang Q, Xue W, Zeng S, Zhang Z, Zhang X, et al. CXC chemokine receptor 4 (CXCR4) antagonist, a novel pathway to prevent chronic allograft nephropathy. Ann Transplant. 2016 Nov;21:728-34.

52 Al-Hamidi A, Pekalski M, Robertson H, Ali S, Kirby JA. Renal allograft rejection: the contribution of chemokines to the adhesion and retention of alphaE(CD103)beta7 integrin-expressing intratubular T cells. Mol Immunol. 2008 Sep;45(15):4000-7.

53 Gao C, Huan J. SDF-1 plays a key role in chronic allograft nephropathy in rats. Transplant Proc. 2008 Jun;40(5):1674-8.

54 Cao Z, Zhang G, Wang F, Liu H, Liu L, Han Y, et al. Protective effects of mesenchymal stem cells with CXCR4 up-regulation in a rat renal transplantation model. PLoS One. 2013 Dec; 8(12):e82949.

55 Ratajczak MZ, Lee H, Wysoczynski M, Wan W, Marlicz W, Laughlin MJ, et al. Novel insight into stem cell mobilization-plasma sphingosine-1-phosphate is a major chemoattractant that directs the egress of hematopoietic stem progenitor cells from the bone marrow and its level in peripheral blood increases during mobilization due to activation of complement cascade/membrane attack complex. Leukemia. 2010 May;24(5):976-85.

56 de Vivar Chevez AR, Finke J, Bukowski R. The role of inflammation in kidney cancer. Adv Exp Med Biol. 2014;816:197-234.

57 Lee JP, Bae JB, Yang SH, Cha RH, Seong EY, Park YJ, et al. Genetic predisposition of donors affects the allograft outcome in kidney transplantation; polymorphisms of stromal-derived factor-1 and CXC receptor 4. PLoS One. 2011 Feb;6(2):e16710.

58 Wang CJ, Tsai JP, Yang SF, Lian JD, Chang HR Stromal cell-derived factor 1 gene polymorphism is associated with susceptibility to adverse long-term allograft outcomes in non-diabetic kidney transplant recipients. Int J Mol Sci. 2014 Jul;15(7):12495-506.

59 Pan J, Mestas J, Burdick MD, Phillips RJ Thomas GV, Reckamp K, et al. Stromal derived factor-1 (SDF-1/CXCL12) and CXCR4 in renal cell carcinoma metastasis. Mol Cancer. 2006 Nov;5:56.

60 Struckmann K, Mertz K, Steu S, Storz M, Staller P, Krek W, et al. pVHL co-ordinately regulates CXCR4/CXCL12 and MMP2/MMP9 expression in human clear-cell renal cell carcinoma. J Pathol. 2008 Mar;214(4):464-71.

$61 \mathrm{Wu} F, \mathrm{Wu}$ S, Gou X. Identification of biomarkers and potential molecular mechanisms of clear cell renal cell carcinoma. Neoplasma. 2018;65(2):242-52.

62 Wang J, Yuan L, Liu X, Wang G, Zhu Y, Qian $\mathrm{K}$, et al. Bioinformatics and functional analyses of key genes and pathways in human clear cell renal cell carcinoma. Oncol Lett. 2018 Jun 15(6):9133-41.
63 Cai C, Wang LH, Dong Q, Wu ZJ, Li MY, Sun $\mathrm{YH}$. Association of CXCL12 and CXCR4 gene polymorphisms with the susceptibility and prognosis of renal cell carcinoma. Tissue Antigens. 2013 Sep;82(3):165-70.

64 Gahan JC, Gosalbez M, Yates T, Young EE, Escudero DO, Chi A, et al. Chemokine and chemokine receptor expression in kidney tumors: molecular profiling of histological subtypes and association with metastasis. J Urol. 2012 Mar; 187(3):827-33.

65 Schrader AJ, Lechner O, Templin M, Dittmar KE, Machtens S, Mengel M, et al. CXCR4/ CXCL12 expression and signalling in kidney cancer. Br J Cancer. 2002 Apr;86(8):1250-6.

66 Wang L, Chen W, Gao L, Yang Q, Liu B, Wu Z, et al. High expression of CXCR4, CXCR7 and SDF-1 predicts poor survival in renal cell carcinoma. World J Surg Oncol. 2012 Oct;10:212.

67 Wehler TC, GrafC, Altherr K, Zimmermann T, Brenner W, Thüroff JW, et al. SDF1 $\beta$ expression in renal cell carcinoma correlates with grading and infiltration by CD8+ T-cells. Anticancer Res. 2011 Sep;31(9):2797-803.

68 Rasti A, Abolhasani M, Zanjani LS, Asgari M, Mehrazma M, Madjd Z. Reduced expression of CXCR4, a novel renal cancer stem cell marker, is associated with high-grade renal cell carcinoma. J Cancer Res Clin Oncol. 2017 Jan: 143(1):95-104.

69 Tang B, Tang F, Li Y, Yuan S, Li B, Wang Z, et al. Clinicopathological significance of CXCR4 expression in renal cell carcinoma: a meta-analysis. Ann Surg Oncol. 2015 Mar;22(3):1026-31.

70 Floranović MP, VeličkovićLJ.Effect of CXCL12 and its receptors on unpredictable renal cell carcinoma. Clin Genitourin Cancer. 2020 Aug; 18(4):e337-42.

71 Wang L, Wang L, Yang B, Yang Q, Qiao S, Wang Y, et al. Strong expression of chemokine receptor CXCR4 by renal cell carcinoma cells correlates with metastasis. Clin Exp Metastasis. 2009;26(8):1049-54.

72 Wang L, Wang Z, Yang B, Yang Q, Wang L, Sun Y. CXCR4 nuclear localization follows binding of its ligand SDF-1 and occurs in metastatic but not primary renal cell carcinoma. Oncol Rep. 2009 Dec;22(6):1333-9.

73 Jones J, Marian D, Weich E, Engl T, Wedel S, Relja B, et al. CXCR4 chemokine receptor engagement modifies integrin dependent adhesion of renal carcinoma cells. Exp Cell Res. 2007 Nov;313(19):4051-65.

74 Wang L, Huang T, Chen W, Gao X, Zhou T, $\mathrm{Wu} \mathrm{Z}$, et al. Silencing of CXCR4 by RNA interference inhibits cell growth and metastasis in human renal cancer cells. Oncol Rep. 2012 Dec; 28(6):2043-8.

75 Tarasova NI, Stauber RH, Michejda CJ. Spontaneous and ligand-induced trafficking of CXC-chemokine receptor 4. J Biol Chem. 1998 Jun;273(26):15883-6.

76 Gassenmaier M, Chen D, Buchner A, Henkel L, Schiemann M, Mack B, et al. CXC chemokine receptor 4 is essential for maintenance of renal cell carcinoma-initiating cells and predicts metastasis. Stem Cells. 2013 Aug;31(8):1467-76. 